preprint BA-98-45

October 1998

\title{
Implications of HERA results for very high energy cosmic ray physics*
}

\author{
Ralph Engel a \\ ${ }^{a}$ University of Delaware, Bartol Research Institute, Newark, DE 19716 USA, e-mail: \\ eng@lepton.bartol.udel.edu
}

Experimental results obtained with the HERA collider and recent progress in their theoretical interpretation are reviewed. After a short introduction to HERA physics, deep inelastic scattering and photoproduction are discussed as (virtual) photon-proton scattering. It is shown that the measurement and theoretical understanding of both photoproduction as well as low- $x$ deep inelastic scattering are essential for obtaining reliable high energy extrapolations within hadron-hadron interaction models. Limitations of the predictive power of hadron interaction models due to the interplay of perturbative QCD and unitarity effects are discussed.

\section{Introduction}

It is very difficult to compare data measured at the HERA collider directly to data obtained in cosmic ray experiments. Almost all cosmic ray experiments deal with final state particles (hadrons, electrons, photons, muons, or neutrinos) which are produced in interactions of primary cosmic ray (CR) particles with air. Furthermore, the experiments are very sensitive to the projectile fragmentation region. In the contrary, modern collider experiments focus on particle production in the central pseudorapidity range in the projectile-target center-of-mass system (CMS). They either deal with leptons, (anti)protons or heavy nuclei as primaries. Nevertheless, it is possible to relate particle production processes in CR interactions to those studied in collider experiments.

Since the number of final state particles is often very large, efficient approximations as well as simulation techniques have to be applied to derive conclusions from measured data or to study theoretical predictions. One of the most powerful technique is the realization of theoretical models in Monte Carlo event generators. Such event generators allow us to study hadron production at colliders as well as in $\mathrm{CR}$ interactions.

\footnotetext{
*Invited talk given at the Xth Int. Symposium on Very High Energy Cosmic Ray Interactions, Gran Sasso, July 12-17, 1998.
}

Unfortunately, our current understanding of hadronic interaction processes is rather limited. Therefore, these event generators can only be built by combining theoretical predictions with phenomenological models and parametrizations and have to be tuned by comparing their predictions to fixed target and collider measurements. Considering the underlying theory and models entering such Monte Carlo programs, almost all data measured at HERA are relevant to our understanding of very high energy cosmic ray interactions. Examples include data on

(i) limits on physics beyond the Standard Model, (ii) parton densities,

(iii) reliability and range of applicability of perturbative QCD and various approximation schema,

(iv) transition between soft and hard physics (perturbative and non-perturbative regimes),

(v) forward hadron production, and

(vi) heavy flavour production.

It is beyond the scope of this work to discuss all these data. Instead, we shall focus on the implications of the low- $x$ structure function measurements [1] 近 and recent results on forward jet [5, and leading baryon production [7,8. Where possible, comparisons to predictions of Monte Carlo models currently used in the analysis of CR data are included.

After a short introduction to the HERA collider, deep inelastic scattering (DIS) and parton 
densities at low $x$ are discussed in Sec. 3. In Sec. A DIS is interpreted as (virtual) photonproton scattering and data on leading jet and baryon production are presented. Finally, in Sec. 5 a summary is given.

\section{The HERA collider}

The electron-proton storage ring HERA at DESY, Hamburg (Germany) went into operation in 1992. At HERA, 27.6 GeV positrons or electrons are collided on $820 \mathrm{GeV}$ protons. The leptonf-proton c.m. energy is $\sqrt{s}_{e p} \approx 300 \mathrm{GeV}$ which corresponds to a lepton energy of $E_{\text {lab }} \approx 47 \times 10^{12}$ $\mathrm{eV}$ in the proton rest frame.

The typical scattering process studied at HERA is sketched in Fig. 1. The beam lepton

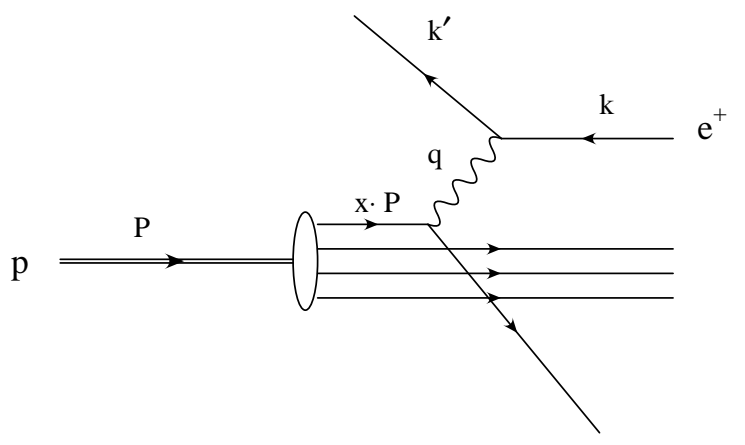

Figure 1. Kinematics of positron-proton scattering in Breit frame.

scatters off the proton by exchanging a photon (with a small cross section, the exchanged particle might be also a $W^{ \pm}, Z$ gauge boson). The kinematics of the reaction can be characterized by the following quantities

- photon virtuality: $Q^{2}=-q^{2}=-\left(k-k^{\prime}\right)^{2}$

- Bjorken's scaling variable: $x=Q^{2} /(2 P \cdot q)$

- inelasticity: $y=P \cdot q /(P \cdot k)$.

${ }^{2}$ Thereafter the term 'lepton' is used to refer either to electrons or positrons accelerated at HERA.
There are only two independent variables, we shall use $x$ and $Q^{2}$. In the proton infinitemomentum frame, $x$ denotes the momentum fraction of the proton carried by the struck quark.

In Fig. 2 the kinematic range for DIS accessible by various fixed-target experiments and HERA is shown. The HERA measurements extend the

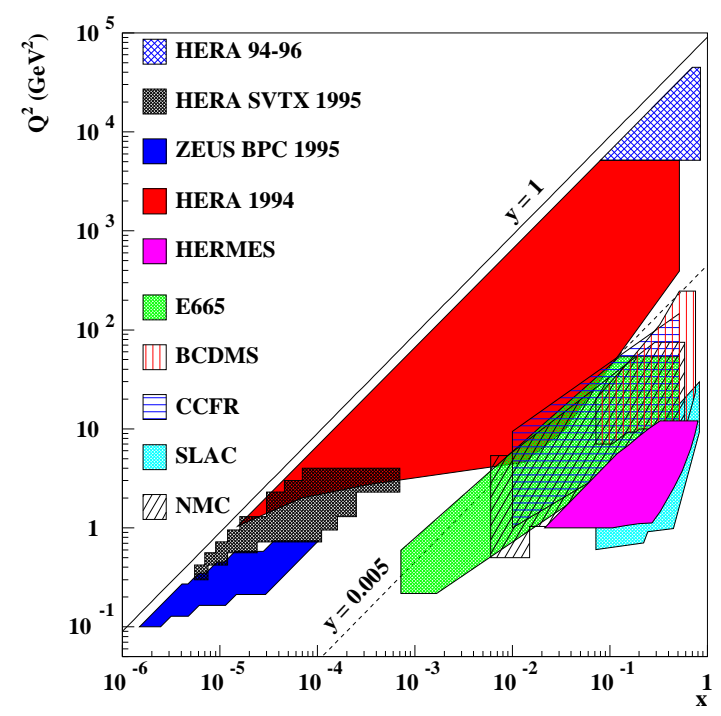

Figure 2. Kinematic region of DIS covered by experiments up to now 9 .

previously explored kinematical range by about two orders in magnitude in $x$ and $Q^{2}$.

\section{Structure function and low- $x$ parton densities}

The structure functions $F_{2}$ and $F_{L}$ are defined by the differential lepton-proton cross section

$$
\begin{aligned}
\frac{d^{2} \sigma_{e p}}{d x d Q^{2}}= & \frac{2 \pi \alpha^{2}}{Q^{4} x}\left[\left(1+(1-y)^{2}\right) F_{2}\left(x, Q^{2}\right)\right. \\
& \left.-y^{2} F_{L}\left(x, Q^{2}\right)\right]
\end{aligned}
$$

where $\alpha$ denotes the fine structure constant. For most applications the longitudinal structure function $F_{L}$ can be neglected since $y$ is sufficiently small. 
In leading order perturbative QCD the structure function $F_{2}$ is related to the quark densities $q, \bar{q}$ in the proton by

$$
F_{2}\left(x, Q^{2}\right)=x \sum_{i} e_{i}^{2}\left(q_{i}\left(x, Q^{2}\right)+\bar{q}_{i}\left(x, Q^{2}\right)\right),
$$

where $e_{i}$ is fractional charge of the quark $i$. The Dokshitzer-Gribov-Lipatov-AltarelliParisi (DGLAP) equations 10 12] predict the $Q^{2}$ dependence of the quark and gluon densities (for large $Q^{2}$ and not too small $x$ )

$$
\begin{aligned}
& \frac{\partial}{\partial \ln Q^{2}}\left(\begin{array}{c}
q\left(x, Q^{2}\right) \\
g\left(x, Q^{2}\right)
\end{array}\right) \\
& \quad=\frac{\alpha_{s}\left(Q^{2}\right)}{2 \pi}\left(\begin{array}{ll}
P_{q q} & P_{q g} \\
P_{g q} & P_{g g}
\end{array}\right) \otimes\left(\begin{array}{c}
q\left(x, Q^{2}\right) \\
g\left(x, Q^{2}\right)
\end{array}\right) .
\end{aligned}
$$

Here $P_{i j}$ represents the splitting kernel describing the probability of finding a daughter parton $i$ in the parent parton $j$ (e.g. $\sum_{k} P(j \rightarrow$ $i, k)$ ) and we have used $\alpha_{s}=g_{s}^{2} /(4 \pi)$ with $g_{s}$ being the strong coupling constant. In the limit $\ln (1 / x), \ln \left(Q^{2} / \Lambda^{2}\right) \rightarrow \infty$ (double leading-logarithmic approximation) the DGLAP equations predict a steeply rising gluon density $g\left(x, Q^{2}\right)$

$$
\begin{aligned}
x g\left(x, Q^{2}\right) & \sim \exp \left[\frac{48}{11-\frac{2}{3} n_{f}} \ln \frac{\ln \frac{Q^{2}}{\Lambda^{2}}}{\ln \frac{Q_{0}^{2}}{\Lambda^{2}}} \ln \frac{1}{x}\right]^{\frac{1}{2}} \\
& \sim \frac{1}{x^{0.4}}
\end{aligned}
$$

where $\Lambda$ denotes the QCD renormatization scale and $n_{f}$ is the number of quark flavours. Since the gluon and sea quark densities are closely related (see Eq. (3i)), a rise of $F_{2}$ is expected for decreasing $x$. This has been confirmed by H1 and ZEUS measurements. Experimentally, the gluon density can be estimated, for example, from the scaling violation of $F_{2}$ ( $Q^{2}$ dependence) or from the diffractive $J / \Psi$ production cross section. In Fig. 3 the gluon density is shown for $Q^{2}=20 \mathrm{GeV}^{2}$ together with several theoretical predictions. Currently, data on quark and gluon densities are available for $x$ as low as $10^{-4}$ and $Q^{2} \approx 20 \mathrm{GeV}^{2}$. These measurements lead to an improved knowledge of the low- $x$ parton densities which has already been applied in many

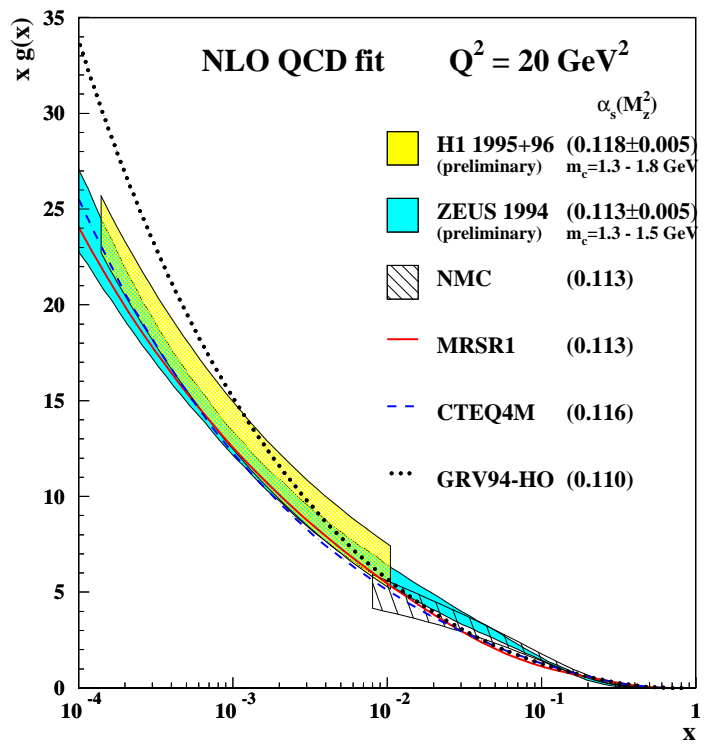

Figure 3. Gluon density at low $x$ and $Q^{2}=20$ $\mathrm{GeV}^{2}$. Shown are H1, ZEUS and NMC data together with several parton density parametrizations.

CR calculations, for example, calculations of neutrino interaction length, charm production, and prompt muon yields 13, 14.

Concerning ultra-high energy CR interactions, it is needed to extrapolate the measured parton densities down to lower $x$ by several orders of magnitude. For example, partons with $x$ values of $10^{-6}$ to $10^{-7}$ dominate minijet and charm production in proton-air interactions at $E_{\mathrm{lab}} \approx 10^{20}$ $\mathrm{eV}$ and are important in the case of neutrino-air interactions. The production of jets with several $\mathrm{GeV}$ transverse momentum in very forward direction involves even smaller $x$ values of about $10^{-10}$.

From the HERA data it is clear that the gluon density rises rapidly at low $x$. It is convenient to parametrize this in terms of the power $\Delta_{H}$ with (see Eq. (5))

$x g\left(x, Q^{2}\right) \sim \frac{1}{x^{\Delta_{H}}}$,

where data suggest $\Delta_{H} \approx 0.25 \ldots 0.4$. However, it is clear from simple geometrical arguments that 
the rapid growth of the gluon density at low $x$ will eventually be tamed by saturation effects [15, 16.

Let's consider the scattering of a quark with the virtuality $Q^{2}$ off a proton. This virtual quark probes an effective transverse area of the size of $\sim \alpha_{s}^{2}\left(Q^{2}\right) / Q^{2}$. As soon as the average transverse distance between the gluons in the proton becomes smaller than $\sim 1 / \sqrt{Q^{2}}$, several gluons participate in a single quark-proton interaction and the effective number of gluons "seen" by the quark is smaller than naively expected. At low $x$ the effective number of gluons saturates with an upper limit of

$x g\left(x, Q^{2}\right) \sim R_{0}^{2} Q^{2}$,

where $R_{0}$ is a measure of the radius of the transverse area available to gluons in the proton. This could be either the proton radius $\left(R_{0}^{2} \sim 5 \mathrm{GeV}^{-2}\right)$ itself or the size of gluon clouds around the valence quarks (hot spot scenario, $R_{0}^{2} \sim 2 \mathrm{GeV}^{-2}$ ), see Fig. A. It is important to note that satura-

democratic distribution:
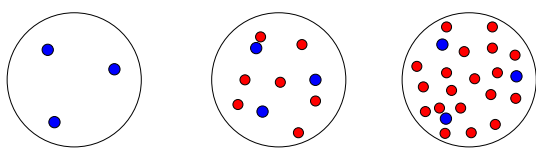

hot spots:

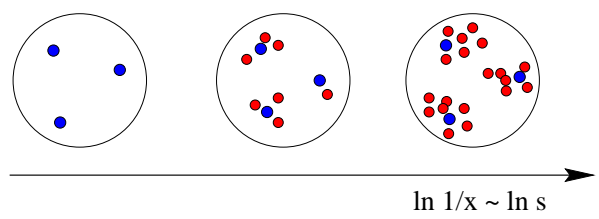

Figure 4. Two possible scenarios for the evolution of the parton density for decreasing $x$.

tion effects might become important already for $x g\left(x, Q^{2}\right) \ll R_{0}^{2} Q^{2}$ as diffractive processes observed in DIS indicate (for example, see [17,18). Indeed, there are theoretical arguments supporting the hypothesis that the gluon density might not reach the saturation limit (7) implied by such simple geometrical considerations (see, for example, 19 21; an overview on high-density parton evolution equations can be found in 22]).

The gluon density is closely linked to the scaling violation of $F_{2}$

$x g\left(x, Q^{2}\right) \simeq \frac{4 \pi}{(40 / 27) \alpha_{s}} \frac{\partial F_{2}\left(\frac{x}{2}, Q^{2}\right)}{\partial \ln Q^{2}}$.

This gives a handle to measure saturation effects. It follows from Eq. (7) that in case of saturation

$\frac{\partial F_{2}}{\partial \ln Q^{2}} \sim R_{0}^{2} Q^{2}$

instead of $\partial F_{2} / \partial \ln Q^{2} \sim x^{-\Delta_{H}}$. In Fig. 5 ZEUS data are shown together with the prediction according to the DGLAP evolution equations (the Glück-Reya-Vogt (GRV) 23] parton densities have been used). Although it is possible to

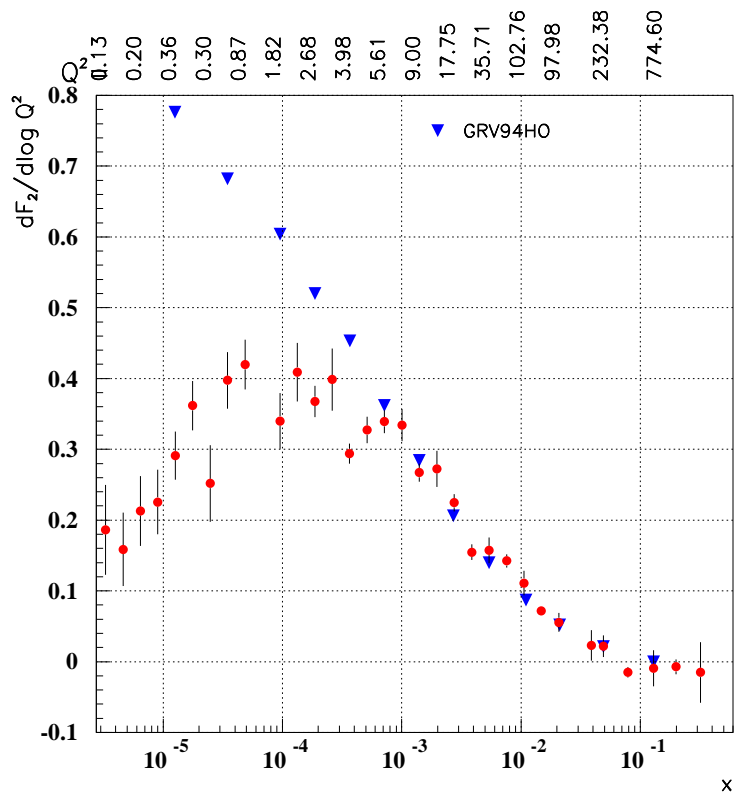

Figure 5. $F_{2}$ scaling violation plotted as function of Bjorken- $x$. ZEUS data (circles) [24] are compared to the GRV prediction [23] (triangles).

fit the data within the DGLAP framework assuming a valence-like gluon density at low $x$ [25,26, 
it is very likely that first hints of parton density saturation have been found [27].

The steep rise and the expected saturation of the gluon density at low $x$ have several implications for the interpretation of $\mathrm{CR}$ interactions. On one hand it is clear that the minijet cross section in hadron-air interactions increases rapidly with $\sqrt{s}$. On the other hand the saturation effects make it very difficult to derive reliable cross sections for all processes where gluon densities at very low $x$ are involved. This applies in particular to the minijet and charm production cross sections in proton-proton and hence in hadronair collisions and also, but to a lesser extent, to ultra-high energy neutrino cross sections.

In most of the hadronic air shower simulation programs the production of minijets is one important ingredient. These Monte Carlo event generators are based on the DGLAP evolution equations (3). Therefore it is interesting to consider the implication of the low- $x$ HERA data for the highenergy extrapolations done within these models. Without referring to some particular model, the HERA data imply that

- the charged particle multiplicity rises with the energy faster than $\ln (s)$ but slower than $s^{\Delta_{H}}$, and

- the mean transverse momentum of hadronic secondaries rises faster than $\ln (s)$.

This can be understood by considering the transverse momentum distribution of hadronic secondaries produced in $p p$ interactions as shown in Fig. 6. The region labeled "hard" is assumed to be calculable on the basis of the QCD-improved parton model. The "soft" region is dominated by saturation effects and cannot be calculated perturbatively at all. The transition region is effected by saturation effects but might still be calculable with perturbative means. For hard processes the differential jet cross section follows from

$$
\frac{d \sigma}{d \eta d p_{\perp}^{2}} \sim \frac{\alpha_{s}^{2}\left(p_{\perp}^{2}\right)}{p_{\perp}^{4}} x_{1} g\left(x_{1}, p_{\perp}^{2}\right) x_{2} g\left(x_{2}, p_{\perp}^{2}\right),
$$

where $x_{1}, x_{2}$ are the momentum fractions of the partons engaged in the hard scattering and $p_{\perp}$ is

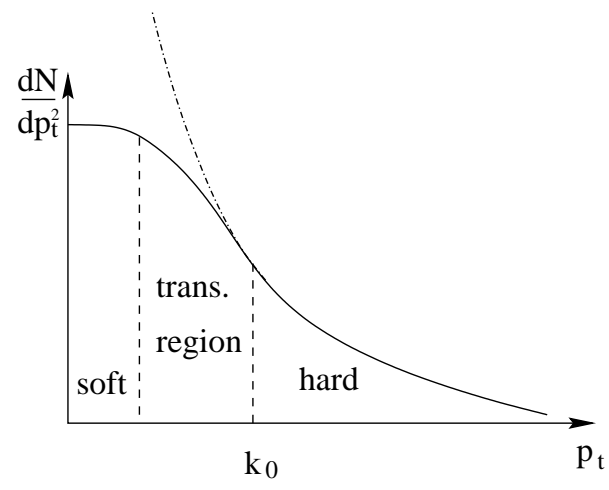

Figure 6. Transverse momentum distribution of hadrons produced in the central pseudorapidity region in CMS.

the jet transverse momentum and $\eta$ denotes the pseudorapidity. After integration over the phase space of the final state particles one finds, see for example 28,

$\sigma\left(p_{\perp} \geq k_{0}\right) \sim s^{\Delta_{H}}$,

for a singular gluon distribution (6). Consequently, the hard part of the cross section rises much faster with the energy than the soft part $\left(\sigma_{\text {soft }} \sim s^{\Delta_{S}}, \Delta_{S}=0.07 \ldots 0.15\right)$. In order to maintain a steady transition between soft and hard processes the value of $k_{0}$ has to increase with the energy (which is also obvious from Eq. (7)). A simple estimate for the parameter $k_{0}$ can be made using 15

$k_{0} \sim \exp \{c \sqrt{\ln s}\}$

which follows from Eqs. (田, (7). The parameter $c$ depends on the assumed low- $x$ behaviour of the gluon density (6). In Fig. 7 the results for $c=0.9$ $\left(\Delta_{H}=0.3\right)$ and $c=1.12\left(\Delta_{H}=0.44\right)$ are shown.

In a DGLAP-based model, realizing steeply rising parton densities at low $x$ but neglecting saturation effects, the average multiplicity of secondaries becomes proportional to the minijet rate at very high energies. Consequently the multiplicity 


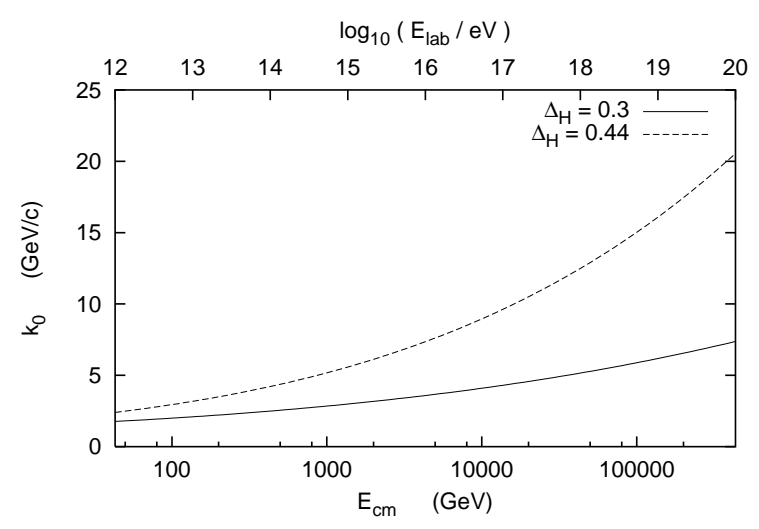

Figure 7. Estimate for the parameter $k_{0}$ characterizing the transverse momentum below which saturation effects should be important.

increases with $s$ according to a power law

$n_{\mathrm{ch}} \sim n_{\mathrm{jet}} \approx \frac{\sigma_{\mathrm{jet}}}{\sigma_{\text {ine }}} \sim \frac{s^{\Delta_{H}}}{s^{0.08}} \sim s^{0.1 \ldots 0.3}$.

The inclusion of saturation effects reduces significantly this rapid increase

$n_{\mathrm{ch}} \sim k_{0}^{2} \sim \exp \{2 c \sqrt{\ln s}\} \quad \gg \ln (s)$

but the multiplicity still grows considerably faster than $\ln (s)$ as implied by limiting fragmentation and Feynman scaling. Hence, HERA data exclude multiplicity extrapolations of the type (13) as found, for example, in QGSJET [29] but also disfavour a pure $\ln (s)$ extrapolation. In Fig. 8 the two limiting extrapolations are shown, the upper curve corresponds to a DGLAP implied power law and the lower curve represents a $\ln (s)$ extrapolation of the collider data.

In the very high energy limit the mean transverse momentum of secondaries is of the order of $k_{0}$. Neglecting saturation effects (which means assuming $k_{0}=$ const.), the mean transverse momentum of secondaries does not increase beyond a certain value even at ultra high energies. By contrast, the inclusion of saturation effects leads to an increase of the average $p_{\perp}$ which is faster than $\ln (s)$. The size of this effect can be seen in Fig. 9. Most of the currently used models show a

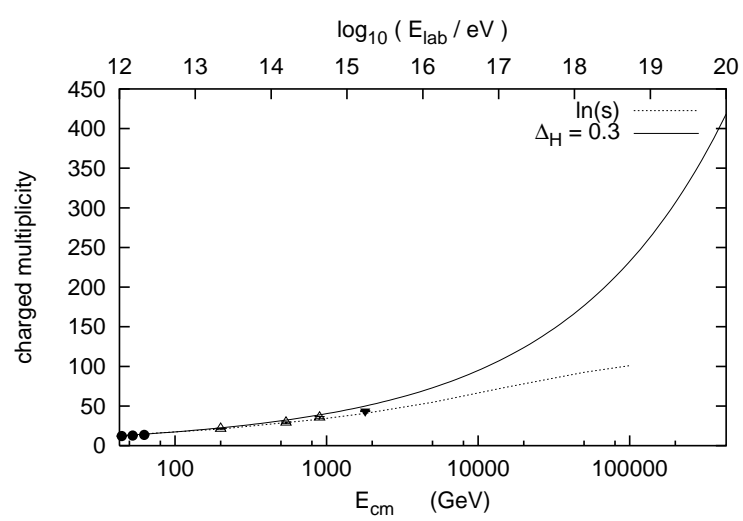

Figure 8. Average charged particle multiplicity in $p p$ collisions. The data are taken from 30 33]

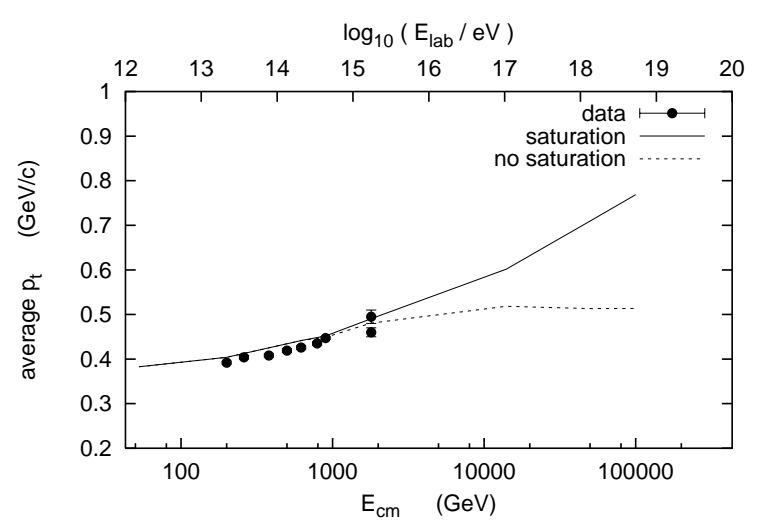

Figure 9. Average transverse momentum of secondaries in $p p$ collisions. Shown are model extrapolations of collider data 34 35 with and without taking into account saturation effects. The calculation has been done with PHOJET [36, 37].

very moderate increase of the average $p_{\perp}$ which is comparable with the dashed curve [38]. Only the DPMJET Monte Carlo 39] predicts a rapid increase of the average transverse momentum similar to the solid curve in Fig. 9. In air shower experiments, the lateral spread of muons with energies of the order of $1 \mathrm{GeV}$ might allow to dis- 
tinguish between the different model predictions.

\section{Hadronic final state in DIS}

Not only structure function data but also the knowledge gained at HERA on the hadronic final state of photon-proton interactions are of interest for CR physics. For example, hard interaction processes are governed by the same parton radiation processes in proton-air interactions and in DIS. As can be seen by comparing Fig. 10 with 11, the parton emissions giving rise to the gluon which couples to the quark in the hard interaction are similar. The emission of partons (the

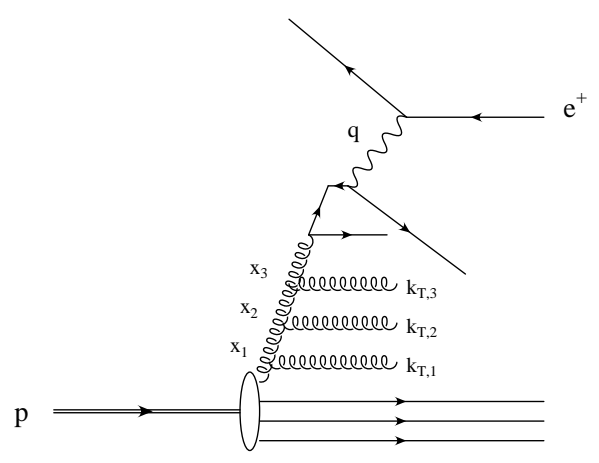

Figure 10. QCD-improved parton model view on DIS.

gluons shown in Figs. 10 and 11) is assumed to obey the DGLAP equations. Since basically almost all perturbative calculations including the event generators used in CR physics are based on these evolution equations, an experimental confirmation is very important ${ }^{3}$ This is in particular true since there exist theoretical arguments that there might be a deviation from the DGLAP predictions for low $x$ processes 40 42]. In the case of DIS the kinematics of the quark loop can be fixed by selecting events with certain scattering angles. By means of measuring the transverse momenta

\footnotetext{
${ }^{3}$ The description of the $Q^{2}$-scaling violation of the structure function $F_{2}$ is an experimental confirmation of the DGLAP equations, however due to the unknown initial conditions not fully conclusive.
}

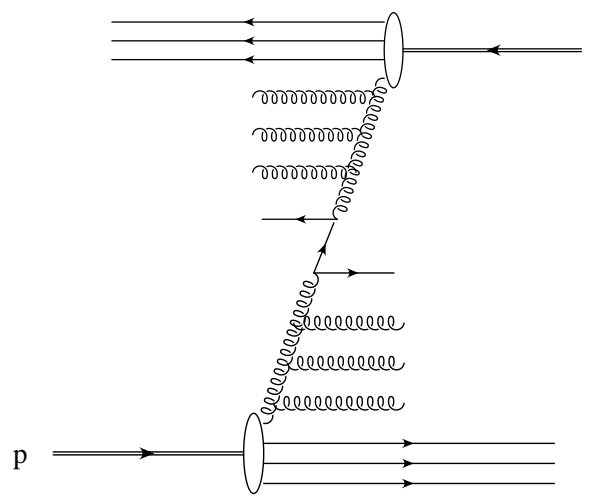

Figure 11. QCD-improved parton model view on jet production in proton-proton scattering.

of the gluon-induced jets $k_{T, 1} \ldots k_{T, 3}$ the predictions of (3) can be tested. The DGLAP evolution equations imply

$$
\begin{aligned}
Q^{2} & \gg k_{T, 3}^{2} \gg k_{T, 2}^{2} \gg k_{T, 1}^{2} \\
& x_{1} \gg x_{2} \gg x_{3} .
\end{aligned}
$$

In Fig. 12 the results of the H1 measurement for forward going jets are shown. Forward-jets roughly correspond to jets produced by the gluon with $k_{T, 1}$ in Fig. 10. The inclusive cross section for these jets with $0.5<k_{T}^{2} / Q^{2}<2$ is plotted as function of the Bjorken- $x$ characterizing the DIS process. Clearly the DGLAP based calculations cannot describe the data. Further measurements are needed to confirm this deviation. There are some important consequences if the DGLAP based approximations break down at low $x$.

- The incoming partons of hard scattering processes have sizable transverse momenta which cannot be neglected.

- Strong $k_{T}$ ordering (15) does not hold at low $x$.

- One parton emission chain can give rise to more than two minijets having almost the same transverse momentum.

The last conclusion can be mathematically rephrased to

$\sigma_{\text {hard }} \neq \frac{1}{2} \int \frac{d \sigma_{\text {jet }}}{d^{2} p_{\perp, \text { jet }}} d^{2} p_{\perp, \text { jet }}$, 


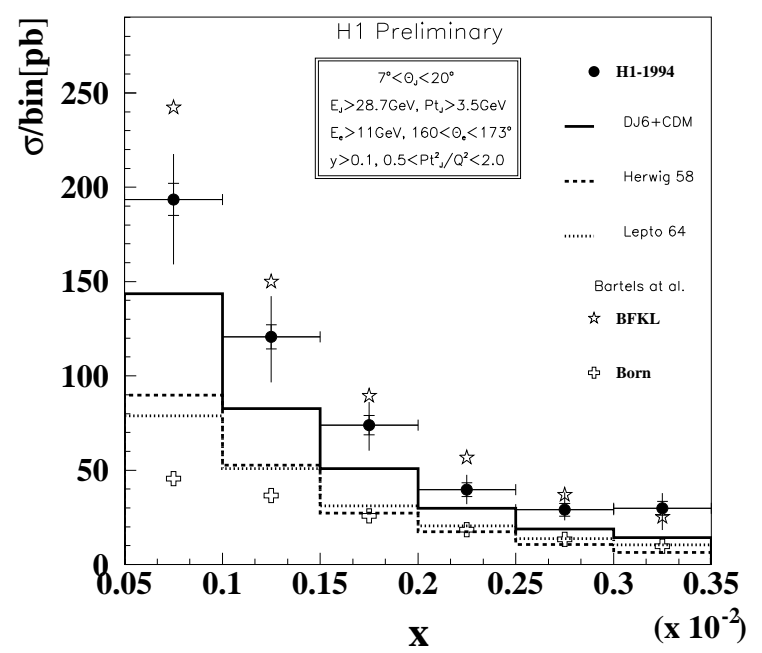

Figure 12. Comparison of $\mathrm{H} 1$ data on forward-jets with theoretical predictions. The dashed and dotted curves correspond to DGLAP based Monte Carlo calculations. The solid curve and the stars represent alternative approaches. For a detailed discussion see [6].

where $\sigma_{\text {hard }}$ is the cross section for hard processes and $d \sigma_{\text {jet }} / d^{2} p_{\perp, \text { jet }}$ is the inclusive jet cross section. Up to now all event generators applied in CR simulations assume that the hard cross section (which is needed for the calculation of the total cross section) is half the inclusive singlejet cross section given by perturbative QCD and collinear factorization (DGLAP approach). The size of the effect has been estimated in [43] and is shown in Fig. 13.

Concerning minimum bias physics, photonproton collisions are very similar to pion-proton or proton-proton interactions. This is well understood in terms of the Vector Dominance Model (see, for example, 44]). The transition form real $\left(Q^{2} \approx 0\right)$ to highly virtual photons changes the event properties. The hadronic final state can be subdivided into photon fragmentation, central, and proton fragmentation regions. As has been confirmed experimentally, hadron production in the proton fragmentation region is almost

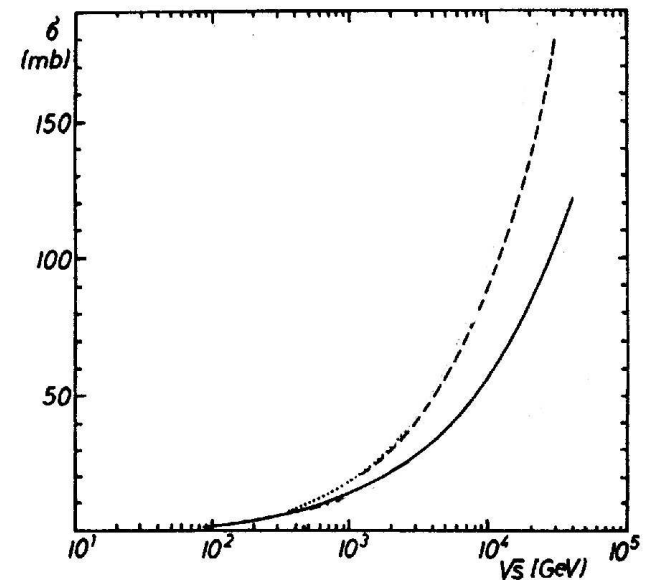

Figure 13. Comparison of the hard cross section as calculated within the collinear factorization approximation (dashed line) and an alternative approach which is expected to be more suited for low- $x$ processes (solid line). The graph is taken from 43 .

independent of the photon virtuality $Q^{2}$. This supports the hypothesis of factorization 45 and allows us to compare data on leading baryon production in $\gamma p$ collisions to Monte Carlo predictions for $p p$ interactions at the same energy. In Fig. 14 the energy fraction $x_{\text {lab }}=E_{\text {baryon }} / E_{\text {beam }}$ carried by the leading proton in photon-proton and proton-proton collisions is shown [7, 8]. There is no $Q^{2}$-dependence of the data within the experimental errors. The data show that there is no dip close to $x_{\text {lab }} \approx 0.9$ as might be expected from the naive application of Feynman scaling violation arguments. The SIBYLl 46,47] and QGSJET [29] models describe the data reasonably well whereas DPMJET 39 seems to underestimate the data close to $x_{\text {lab }} \approx 0.9$.

\section{Summary and outlook}

HERA data and theoretical work related to HERA physics clearly improved our understanding of hadron production in such a way that the uncertainties in the model predictions for very high energy cosmic ray interactions can be re- 


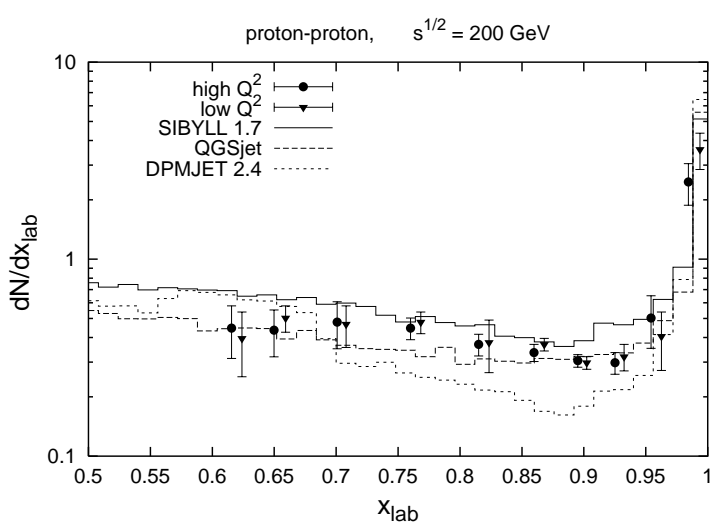

Figure 14. Energy fraction $x_{\text {lab }}$ carried by the leading proton. The data are photoproduction and DIS measurements whereas the curves are model predictions for $p p$ collisions.

duced.

Experimental evidence has been found for a steeply rising gluon density in the proton at low $x$. The inevitable consequence of this finding is that saturation effects are important. First experimental signs for this have been reported. The implication of these results to high-energy extrapolations done within models for $\mathrm{CR}$ interactions include

- the particle multiplicity grows faster than $\ln (s)$ but slower than $s^{\Delta_{H}}$

- the average transverse momentum of secondaries rises faster than $\ln (s)$

Investigations of the hadronic final state in DIS at low $x$ reveal deviations from the prediction obtained in the "standard" DGLAP framework (collinear factorization). Up to now, the physics of hard processes at low $x$ is theoretically not well understood. However, it can be expected that the research activity on this subject triggered by HERA measurements will soon lead to a considerably improved understanding of typical low- $x$ processes such as minijet and charm production.

It has been shown that HERA data can be used to reduce the uncertainties in Monte Carlo model predictions concerning central hadron or jet production as well as leading baryon distributions.

\section{Acknowledgments}

The author acknowledges the excellent collaboration with T. Gaisser, T. Stanev, and P. Lipari on the subject of this work. He is grateful to J. Ranft and S. Roesler for many discussions. The work is supported by the U.S. Department of Energy under Grant DE-FG02-91ER40626.

\section{REFERENCES}

1. H1 Collab.: S. Aid et al.: Nucl. Phys. B470 (1996) 3

2. ZEUS Collab.: M. Derrick et al.: Z. Phys. C72 (1996) 399

3. H1 Collab.: C. Adloff et al.: Nucl. Phys. B497 (1997) 3

4. ZEUS Collab.: J. Breitweg et al.: Phys. Lett. B407 (1997) 432

5. ZEUS Collab.: J. Breitweg et al.: Forward jet production in deep inelastic scattering at HERA, DESY 98-050, (hep-ex/9805016), 1998

6. H1 Collab.: C. Adloff et al.: Forward jet and particle production at HERA, DESY 98-143, (hep-ex/9809028), 1998

7. ZEUS Collab.: A. Garfagnini et al.: Leading baryons at HERA with the ZEUS forward detectors, to appear in the proceedings of the Sixth International Workshop on DeepInelastic Scattering and QCD, Brussels, April 1998

8. W. Schmidke: Leading baryon production in $e p$ scattering at HERA, (in behalf of the $\mathrm{H} 1$ and ZEUS Collaborations), to appear in the proceedings of the XXIXth International Conference on High Energy Physics, Vancouver, Canada, July 1998

9. U. Schneekloth: Recent HERA results and future prospects, DESY 98-060, hepex/9806010), 1998

10. V. N. Gribov and L. N. Lipatov: Sov. J. Nucl. Phys. 15 (1972) 438

11. Y. L. Dokshitzer: Sov. Phys. JETP 46 (1977) 641

12. G. Altarelli and G. Parisi: Nucl. Phys. B126 
(1977) 298

13. R. Gandhi, M. H. Quigg, Reno and I. Sarcevic: Neutrino Interactions at ultrahigh energies, FERMILAB-PUB-98/087-T, hepph/9807264), 1998

14. L. Pasquali, M. H. Reno and I. Sarcevic: Lepton fluxes from atmospheric charm, hepph/9807264), 1998

15. V. N. Gribov, M. L. Levin and M. G. Ryskin: Phys. Rep. 100 (1983) 1

16. E. M. Levin and M. G. Ryskin: Phys. Rep. 189 (1990) 268

17. H1 Collab.: T. Ahmed et al.: Nucl. Phys. B429 (1994) 477

18. ZEUS Collab.: M. Derrick et al.: Phys. Lett. B332 (1994) 228

19. A. Capella, A. Kaidalov, C. Merino and J. Trân Thanh Vân: Phys. Lett. B337 (1994) 358

20. A. Capella, A. Kaidalov, C. Merino, D. Pertermann and J. Trân Thanh Vân: Phys. Rev. D53 (1996) 2309

21. L. Frankfurt and M. Strikman: Phys. Lett. B382 (1996) 6

22. E. M. Levin: Evolution equations for high parton density QCD, TAUP 2506-98, hep$\mathrm{ph} / 9806434), 1998$

23. M. Glück, E. Reya and A. Vogt: Z. Phys. C67 (1995) 433

24. ZEUS Collab.: A. T. Doyle et al.: Highlights and open questions from ZEUS, GLASPPE/98-03, (hep-ex/9808022), to appear in the proceedings of the Sixth International Workshop on Deep-Inelastic Scattering and QCD, Brussels, April 1998

25. A. D. Martin, R. G. Roberts, W. J. Stirling and R. S. Thorne: Eur. Phys. J. C4 (1998) 463

26. ZEUS Collab.: J. Breitweg et al.: ZEUS results on the measurement and phenomenology of $F_{2}$ at low $x$ and low $Q^{2}$, DESY-98-121, (hep-ex/9809005), 1998

27. J. Ellis: Summary of DIS 98, CERN-TH/98203, (hep-ph/9806517), to appear in the proceedings of the Sixth International Workshop on Deep-Inelastic Scattering and QCD, Brussels, April 1998

28. J. Kwieciński and A. D. Martin: Phys. Rev.
D43 (1991) 1560

29. N. N. Kalmykov, S. Ostapchenko and A. I. Pavlov: Nucl. Phys. B (Proc. Suppl.) 52B (1997) 17

30. ABCDHW Collab.: A. Breakstone et al.: Phys. Rev. D30 (1984) 528

31. UA5 Collab: R. E. Ansorge et al.: Z. Phys. C43 (1989) 357

32. UA5 Collab.: G. J. Alner et al.: Phys. Lett. B160 (1985) 193, 199

33. E735 Collab.: C. S. Lindsey et al.: Nucl. Phys. A544 (1992) 343

34. UA1 Collab.: C. Albajar et al.: Nucl. Phys. B335 (1990) 261

35. CDF Collab.: F. Abe et al.: Phys. Rev. Lett. 61 (1988) 1819

36. R. Engel: Z. Phys. C66 (1995) 203

37. R. Engel and J. Ranft: Phys. Rev. D54 (1996) 4244

38. J. Knapp, D. Heck and G. Schatz: Comparison of hadronic interaction models used in air shower simulations and of their influence on shower development and obsevables, FZKA 5828, Wissenschaftliche Berichte, Forschungszentrum Karlsruhe, 1996

39. J. Ranft: Phys. Rev. D51 (1995) 64

40. E. A. Kuraev, L. N. Lipatov and V. S. Fadin: Sov. Phys. JETP 44 (1976) 443

41. E. A. Kuraev, L. N. Lipatov and V. S. Fadin: Sov. Phys. JETP 45 (1977) 199

42. Y. Y. Balitsky and L. N. Lipatov: Sov. J. Nucl. Phys. 28 (1978) 822

43. J. Kwieciński: Phys. Lett. B184 (1987) 386

44. A. Donnachie and G. Shaw: Generalized Vector Dominance, Plenum Press, New York, 1978 in: Electromagnetic Interactions of Hadrons, Volume 2, ed. by A. Donnachie and G. Shaw, p. 169

45. J. D. Bjorken and J. Kogut: Phys. Rev. D8 (1973) 1341

46. J. Engel, T. K. Gaisser, P. Lipari and T. Stanev: Phys. Rev. D46 (1992) 5013

47. R. S. Fletcher, T. K. Gaisser, P. Lipari and T. Stanev: Phys. Rev. D50 (1994) 5710 